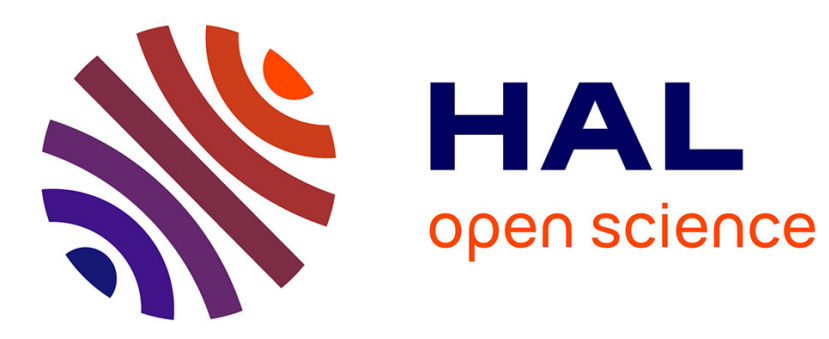

\title{
Merging of two helical vortices
}

Ivan Delbende, Benjamin Piton, Maurice Rossi

\section{To cite this version:}

Ivan Delbende, Benjamin Piton, Maurice Rossi. Merging of two helical vortices. European Journal of Mechanics - B/Fluids, 2015, 49 (B), pp.363-372. 10.1016/j.euromechflu.2014.04.005 . hal-01093398

\section{HAL Id: hal-01093398 https://hal.sorbonne-universite.fr/hal-01093398}

Submitted on 10 Dec 2014

HAL is a multi-disciplinary open access archive for the deposit and dissemination of scientific research documents, whether they are published or not. The documents may come from teaching and research institutions in France or abroad, or from public or private research centers.
L'archive ouverte pluridisciplinaire HAL, est destinée au dépôt et à la diffusion de documents scientifiques de niveau recherche, publiés ou non, émanant des établissements d'enseignement et de recherche français ou étrangers, des laboratoires publics ou privés. 


\title{
Merging of Two Helical Vortices
}

\author{
Ivan Delbende ${ }^{a, b, *}$, Benjamin Piton ${ }^{a, b}$, Maurice Rossi ${ }^{a, c}$ \\ a Sorbonne Universités, UPMC Univ Paris 06, UFR919, F-75005 Paris, France \\ ${ }^{b}$ LIMSI-CNRS, BP 133, F-91403 Orsay Cedex, France \\ ${ }^{c}$ CNRS,UMR 7190, d'Alembert-UPMC, F-75005 Paris, France
}

\begin{abstract}
Vortex merging is a basic fluid phenomenon which has been much studied for two-dimensional flows. Here we extend such a study to a specific class of three-dimensional flows, namely to vortices possessing a helical symmetry. In addition to the standard Reynolds number, this introduces another dimensionless control number, the pitch, which quantifies the periodicity length along the helix axis. Helical vortices with large pitches merge very much as in a two-dimension setting. However, their rotation speed is reduced and the merging period is delayed. These effects, caused by the presence of a self-induction velocity in curved three-dimensional vortices, are understood by computing the streamfunction in the frame of reference rotating with the two vortices, and by inspecting the locations of its hyperbolic points. At intermediate pitch values, only viscous diffusion acts, resulting in a slow viscous type of merging. Finally for small pitches, the system is unstable resulting, at the nonlinear stage, in a different type of merging which breaks the initial central symmetry.
\end{abstract}

Keywords: Vortex, helical vortex, vortex merging, vortex dynamics.

\section{Introduction}

In the wake of propellers [1], wind turbines [2] or helicopters [3], a complex system of vortices is shed possessing the features of general three-dimensional flows: 1) multiple interacting vortices and 2) each vortex being characterised by curvature and torsion. These flows however are somewhat simpler: at least

5 locally, they satisfy a helical symmetry, i.e. these vortices are invariant through combined axial translation and rotation. Many works have already been devoted to this specific class of flows. Steady rotating states

${ }^{*}$ Corresponding author
Email address: Ivan. Delbende@ limsi.fr (Ivan Delbende $\left.{ }^{a, b, *}\right)$ 
vortices have been described in analytical [4] and numerical [5] works, but they are mostly restricted to inviscid filaments and patches.

In this three-dimensional helical context [6], the process of vortex merging is of importance. For in-

\section{Governing equations}

\subsection{Helical symmetry for incompressible flows}

The vortices shed in the wake of a two-blade propeller are at least locally helically symmetric. As a simplifying hypothesis, we will assume the flow to strictly satisfy helical symmetry of helix pitch $2 \pi L$ along a given axis. This means that the velocity field is invariant under the combination of an axial translation of $\Delta z$ and a rotation of angle $\Delta z / L$ around the same axis (figure 1). The flow characteristics are hence identical along the helical lines $\theta-z / L=$ const. Positive (resp. negative) $L$ values correspond to right-handed (resp. left-handed) helices. A scalar field is helically symmetric if it depends on only two space variables $r$ and $\varphi \equiv \theta-z / L$. Helical symmetry for a vector field $\boldsymbol{u}$ can be expressed as follows:

$$
\boldsymbol{u}=u_{r}(r, \varphi, t) \boldsymbol{e}_{r}(\theta)+u_{\varphi}(r, \varphi, t) \boldsymbol{e}_{\varphi}(r, \theta)+u_{B}(r, \varphi, t) \boldsymbol{e}_{B}(r, \theta)
$$


where the orthonormal Beltrami basis (see figure 1) is defined as

$$
\boldsymbol{e}_{B}(r, \theta)=\alpha(r)\left[\boldsymbol{e}_{z}+\frac{r}{L} \boldsymbol{e}_{\theta}(\theta)\right], \quad \boldsymbol{e}_{r}(\theta), \quad \boldsymbol{e}_{\varphi}(r, \theta)=\boldsymbol{e}_{B} \times \boldsymbol{e}_{r}
$$

with $\alpha(r) \equiv\left(1+r^{2} / L^{2}\right)^{-1 / 2}$ taking values ranging from 1 at the axis to 0 at infinite radius.

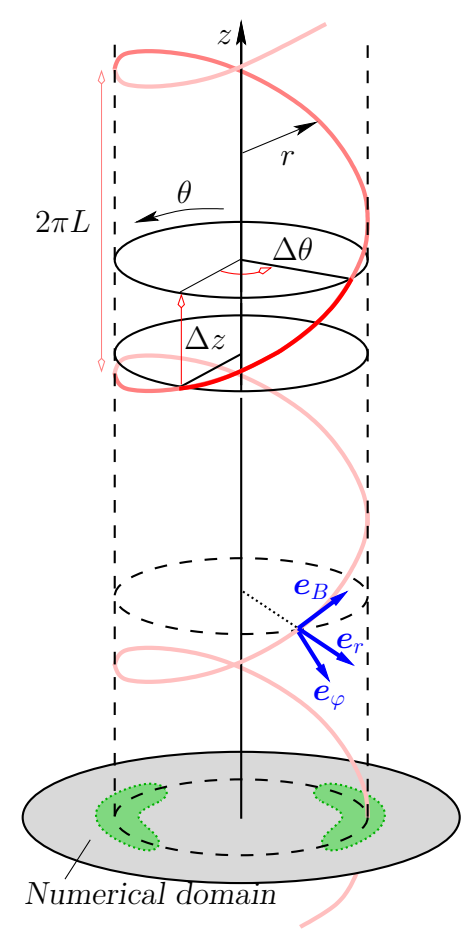

Figure 1: Local helical basis.

In the above framework, a general incompressible helical flow can be expressed as

$$
\boldsymbol{u}=u_{B}(r, \varphi, t) \boldsymbol{e}_{B}+\alpha(r) \nabla \psi(r, \varphi, t) \times \boldsymbol{e}_{B}
$$

where $\psi(r, \varphi, t)$ is a streamfunction providing the two components $u_{r}(r, \varphi, t)$ and $u_{\varphi}(r, \varphi, t)$. In addition, the vorticity field reads

$$
\boldsymbol{\omega}=\omega_{B}(r, \varphi, t) \boldsymbol{e}_{B}+\alpha \nabla\left(\frac{u_{B}(r, \varphi, t)}{\alpha}\right) \times \boldsymbol{e}_{B} .
$$

40 Hence the total flow field is given by two scalar fields, namely $\omega_{B}(r, \varphi, t)$ the vorticity component along the 
unit vector $\boldsymbol{e}_{B}$ and $u_{B}(r, \boldsymbol{\varphi}, t)$ the velocity component along the same vector. Indeed the streamfunction $\psi$ is slaved to both $\omega_{B}$ and $u_{B}$ by equation

$$
\omega_{B}-\frac{2 \alpha^{2}}{L} u_{B}=-\mathbb{L} \psi
$$

where the linear operator $\mathbb{L}$ is a generalised Laplace operator:

$$
\mathbb{L}(\cdot)=\frac{1}{r \alpha} \frac{\partial}{\partial r}\left(r \alpha^{2} \frac{\partial}{\partial r}(\cdot)\right)+\frac{1}{r^{2} \alpha} \frac{\partial^{2}}{\partial \varphi^{2}}(\cdot) .
$$

Using expression (2) the helical component of velocity is written as $u_{B}(r, \varphi, t)=\alpha\left(u_{z}+r u_{\theta} / L\right)$. At large radial distances, the ratio $u_{B}(r, \varphi, t) / \alpha$ thus tends towards the constant $U_{z}^{\infty}+\Gamma /(2 \pi L)$, with $U_{z}^{\infty}=u_{z}(r=\infty)$ and $\Gamma$ the total circulation in the fluid domain. Instead of $u_{B}(r, \varphi, t)$, it is thus convenient to use the quantity

$$
u_{H} \equiv \frac{u_{B}}{\alpha}-\left(U_{z}^{\infty}+\frac{\Gamma}{2 \pi L}\right)
$$

which tends towards 0 when $r \rightarrow \infty$. Up to a multiplicative factor, variable $u_{H}$ corresponds to a longitudinal velocity or jet profile in the vortex core.

\subsection{Navier-Stokes equations with helical symmetry}

The dynamical equations can be thus formulated within a generalization of the standard 2D $\psi-\omega$ method. The equations for $u_{B}$ and $\omega_{B}$ read as

$$
\begin{aligned}
& \partial_{t} u_{B}+N L_{u}=V T_{u} \\
& \partial_{t} \omega_{B}+N L_{\omega}=V T_{\omega}
\end{aligned}
$$

where the nonlinear terms and the viscous terms are given by

$$
\begin{array}{rlrl}
N L_{u} & \equiv \boldsymbol{e}_{B} \cdot[\boldsymbol{\omega} \times \boldsymbol{u}], & V T_{u} & \equiv v\left[\mathbb{L}\left(\frac{u_{B}}{\alpha}\right)-\frac{2 \alpha^{2}}{L} \omega_{B}\right] . \\
N L_{\omega} \equiv \boldsymbol{e}_{B} \cdot \nabla \times[\boldsymbol{\omega} \times \boldsymbol{u}], & V T_{\boldsymbol{\omega}} \equiv v\left[\mathbb{L}\left(\frac{\omega_{B}}{\alpha}\right)-\left(\frac{2 \alpha^{2}}{L}\right)^{2} \omega_{B}+\frac{2 \alpha^{2}}{L} \mathbb{L}\left(\frac{u_{B}}{\alpha}\right)\right] .
\end{array}
$$

The variable $\varphi=\theta-z / L$ being $2 \pi$-periodic, the numerical code uses Fourier series along that direction, and second order finite differences in the radial direction. The time advance is performed using second order backward discretisation of the temporal derivative. Nonlinear terms explicitly appear through second order Adams-Bashforth extrapolation whereas the viscous terms are treated implicitly. At the boundary at $r=R_{\text {ext }}$, potential-flow conditions are imposed, so that the simulation mimics the vortex dynamics in a 
radially infinite flow domain. More details can be found in [7, 8].

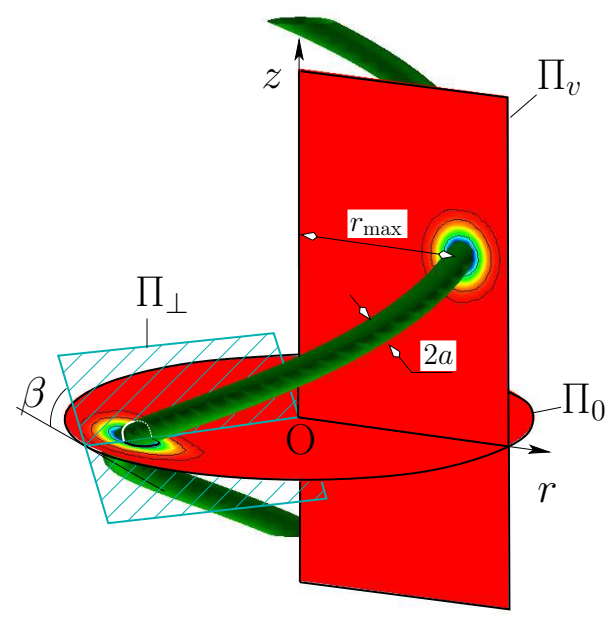

Figure 2: Vortex geometry and characteristic planes.

During simulations, each vortex is characterised by several features (figure 2). First, one locates the point where the maximum helical vorticity $\omega_{B}$ is reached in the plane $z=0$ (denoted hereafter by $\Pi_{0}$ ). This point is determined by its radial $r_{\max }(t)$ and angular position $\theta_{\max }(t)$. This leads to an angular velocity $\Omega(t)=d \theta_{\max } / d t$. Second, the vortex is described by the vorticity $\omega_{B}$ and velocity $u_{H}$ distributions. When visualising the complete vortex system, these quantities are displayed in the $\Pi_{0}$ plane. However, when the characteristics of a single vortex are considered, such fields can be better analyzed in a plane perpendicular to the vortex centerline, so that geometrical deformation effects occurring in the $\Pi_{0}$ plane are eliminated (see figure 2). The plane $\Pi_{\perp}$ is thus introduced, which contains the point $\left(r_{\max }(t), \theta_{\max }(t), z=0\right)$ and is perpendicular to vector $\boldsymbol{e}_{B}^{\max } \equiv \boldsymbol{e}_{B}\left(r_{\max }(t), \theta_{\max }(t)\right)$. Using the invariance of $\omega_{B}$ and $u_{H}$ along helical lines, it is possible to retrieve their values in the plane $\Pi_{\perp}$ from those in the plane $\Pi_{0}$ plane. In analogy to the twodimensional case, a core size $a(t)$ is then defined in the plane $\Pi_{\perp}$ as a dispersion radius [9] of the quantity $\boldsymbol{\omega} \cdot \boldsymbol{e}_{B}^{\max }$.

By choosing adequate initial conditions, the problem can be made generic: one assumes that the flow at $t=0$ results from the time evolution from $t=t_{\star}<0$ of two singular helical vortices of circulation $\Gamma$ located at a radial distance $R_{\star}$ from the axis, and of helical reduced pitch $L$. Clearly the simulation cannot be initiated from this singular filament state. There exists a self-consistent procedure which is not detailed here 
that defines the adequate profile of each vortex: the condition at $t=0$ consists of two identical vortices with an initial thin core $a_{0}$, a pitch $2 \pi L$, and a vorticity maximum located at $\theta_{0}$ and $\theta_{0}+\pi$ in the $\Pi_{0}$ plane. This procedure is based on a two-dimensional ansatz : the singular vortex filaments diffuse in their respective $\Pi_{\perp}$ planes into 2D Gaussian profiles with core sizes $a_{0} \approx \sqrt{-4 v t_{\star}}$. Based on this idea, we choose an initial $\omega_{B}$ distribution in the $\Pi_{0}$ plane such that the associated values of $\omega_{B}$ in the $\Pi_{\perp}$ planes computed through the helicoidal symmetry indeed lead to such Gaussian profiles. The discussion about the initial $u_{H}$ distribution is deferred to section 3.4 (equation 12 ).

In the following, in order to express the evolution from the singular state we always use the time $\tau=t-t_{\star}$ and we adopt the quantities $R_{\star}$ and $R_{\star}^{2} / \Gamma$ as space and time scales. The dynamics is then governed by two dimensionless parameters: the Reynolds number $\operatorname{Re}=\Gamma / v$ and the reduced pitch $\bar{L} \equiv L / R_{\star}$. From now on, all quantities are dimensionless and we drop the bars above the corresponding variables for simplicity.

Simulations are performed in a numerical domain defined by a disk of dimensionless radius $R_{\mathrm{ext}}=3$. It was checked that no significant vorticity reaches the outer boundary, as potential-flow conditions are enforced there. However, for simulations at $L=1.9$ and $L=2$, it was found necessary to move the boundary to $R_{\mathrm{ext}}=4$. The domain is meshed by $N_{r} \times N_{\theta}$ grid points. For Reynolds number $R e=1000$, we choose $N_{r}=256$ and $N_{\theta}=192 ;$ for $R e=2500, N_{r}=384$ and $N_{\theta}=288 ;$ for $R e=5000$ and $10000, N_{r}=512$ and $N_{\theta}=384$.

\section{Merging of large pitch vortices $L \geq 1.9$}

In this section, we consider two helical vortices with a large pitch, typically $L \geq 1.9$, and describe the merging process in that instance.

\subsection{A typical case: $L=2.5, \operatorname{Re}=5000$}

The results plotted in figure 3 are typical for all $L \geq 1.9$ simulations. The vorticity $\alpha \omega_{B}$ and velocity $u_{H}$ components are plotted in the plane $\Pi_{0}$ during a simulation at $L=2.5$ and $R e=5000$. The initial central symmetry is preserved: vortices remain identical and given by a unique radial position $r_{\max }(t)$ and two angular positions $\theta_{\max }(t)$ and $\theta_{\max }(t)+\pi$. Figure 4 provides the value $r_{\max }(t)$ as well as the angular velocity $\Omega(t)$ and core size $a(t)$. In a first phase (snapshot at $\tau=354$ in figures $3 \mathrm{a}$ and $3 \mathrm{~b}$ ), vortices rotate counterclockwise, grow in size through viscous diffusion. During most of the phase, the two vortices slowly drift apart. A second phase of the dynamics follows in which the vortices move towards the center and their angular velocity drastically increases. As there is a continuous shift from phase 1 to phase 2 , it is difficult 
(a)

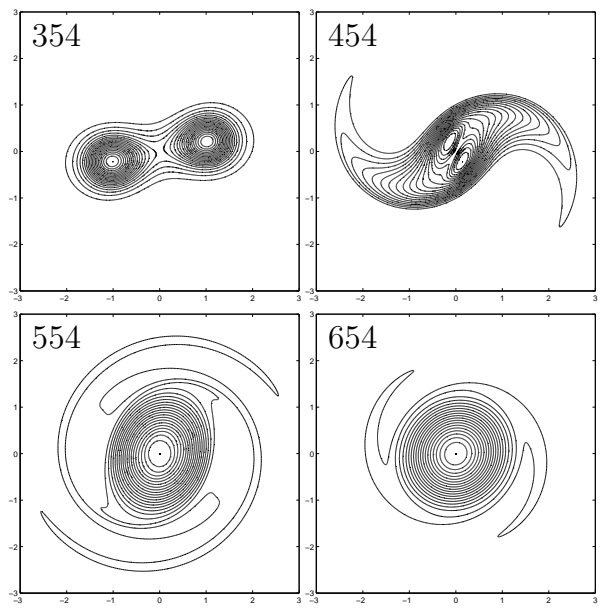

(b)

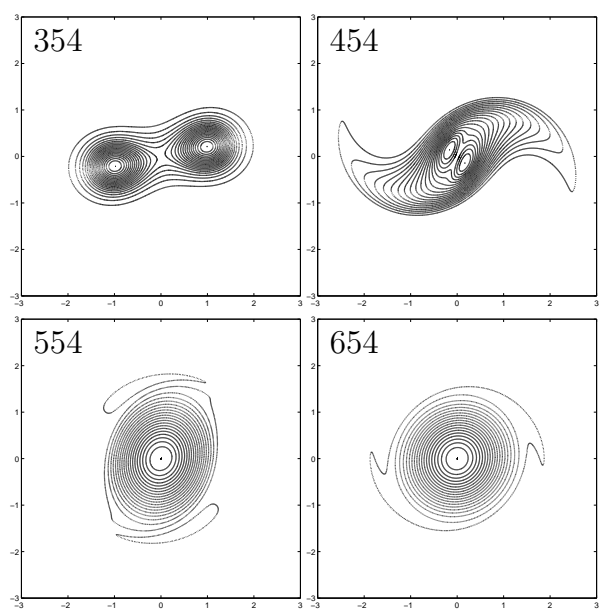

Figure 3: Simulation for $L=2.5$ and $R e=5000$. Isocontours in plane $\Pi_{0}$ of (a) $\alpha \omega_{B}$ (positive values) and (b) $u_{H}$ (negative values) at dimensionless times $\tau=354,454,554,654$.

to define a critical time $\tau_{1}$ for the initiation of phase 2. Here we use the geometrical construction shown on figure 4h to define $\tau_{1}$ (for the specific simulation $\tau_{1}=438$ ). This second phase ends at $\tau_{2}$ where the value $r_{\max }(t)$ reaches a first minimum (for the specific simulation, $\tau_{2}=454$ ). It is followed by a phase characterised by oscillations in which vortices are at a certain distance from the center (see snapshots at $\tau=454$ ). Phase 2 ends at $\tau=\tau_{3}$ when $r_{\max }=0$ (for the specific simulation, $\tau_{3}=529$ ) leading to a single elliptical central vortex with the presence of filaments around the vortex core (snapshot at $\tau=554$ in figures 3 h and $3 \mathrm{~b}$ ). The fourth phase corresponds to the evolution towards the asymptotic diffusing Gaussian state (snapshots at $\tau=654$ in figures 3 and 3 b).

\subsection{Influence of the Reynolds number}

The influence of the Reynolds number on the dynamics is shown on figure 5 for $L=2$. These effects remain valid for all cases with $L \geq 1.9$ :

- Phase 1: the initial diffusion lasts longer as the Reynolds number is increased. It is observed from simulations that the duration $\tau_{1}$ is proportional to a power of $R e$ at a fixed value of $L$ (figure 6). For $L=\infty$, it evolves in $R e$, for $L=2$ in $R e^{0.9}$. The angular velocity $\Omega(\tau)$ does not change with $R e$.

- Phase 2: distance $r_{\max }\left(\tau_{2}\right)$ at the end of phase 2 weakly depends on the Reynolds number (see figure $5 \mathrm{~h}$ ). The duration $\left(\tau_{2}-\tau_{1}\right)$ of this phase has a weak dependence on the Reynolds number: for instance at $L=2,\left(\tau_{2}-\tau_{1}\right) \sim R e^{-0.25}$. 

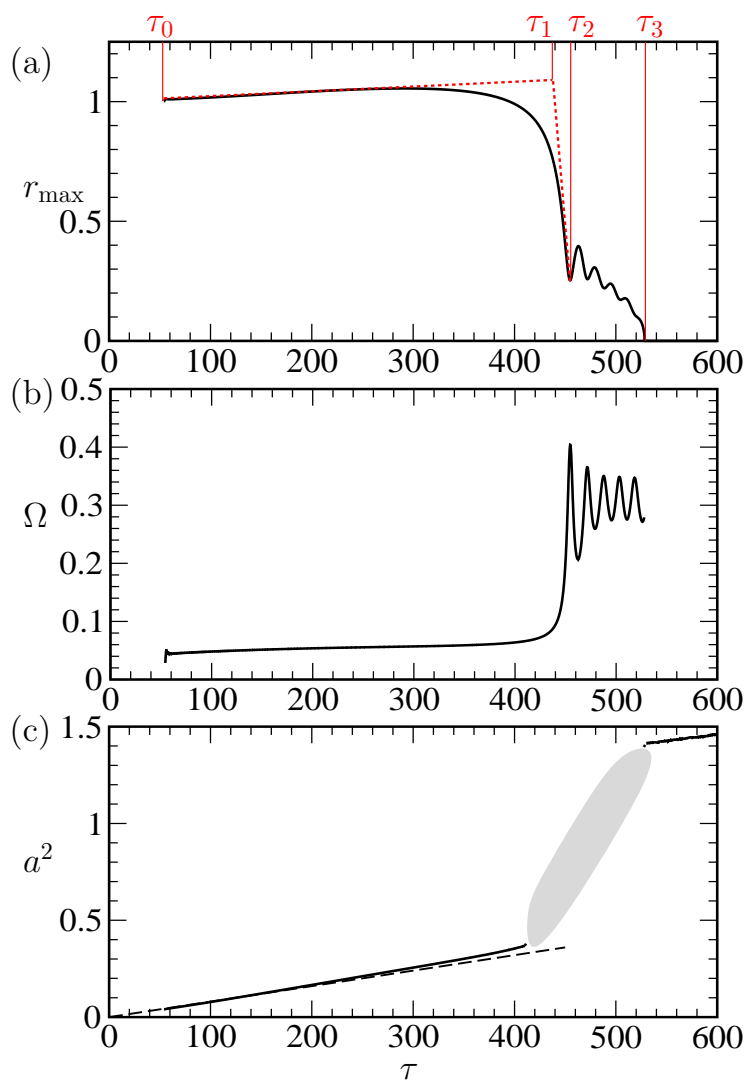

Figure 4: Simulation for $L=2.5$ and $R e=5000$. (a) Radial position $r_{\max }$ of the vorticity maximum as a function of time $\tau$. The geometrical construction shown defines the time $\tau_{1}$, and times $\tau_{2}$ and $\tau_{3}$ are also displayed. (b) Angular velocity $\Omega(\tau)$. (c) Core size $a(\tau)$. During the phases where filamentation takes place, this quantity is not evaluated (shaded region).

- Phase 3: the frequency $\omega_{3}$ of oscillations during phase 3 slightly increases with Reynolds number. The duration $\left(\tau_{3}-\tau_{2}\right)$ of this phase scales as a power of Reynolds number. For $L=\infty$, it evolves in $R e^{0.5}$, for $L=2$ in $R e^{0.78}$.

Finally, as seen in figure 5 , the core size $a^{2}(\tau)$ evolves as a standard two-dimensional diffusion i.e. as $a^{2}(\tau)=4 \tau / \operatorname{Re}$.

\subsection{Influence of the helical pitch: angular velocity and merging}

For large $L \geq 1.9$ values, decreasing the pitch $L$ from $L=\infty$ has a marked slowdown effect on the merging process at constant Reynolds number (figure 7). In particular, the vortices rotate at a weaker angular velocity 

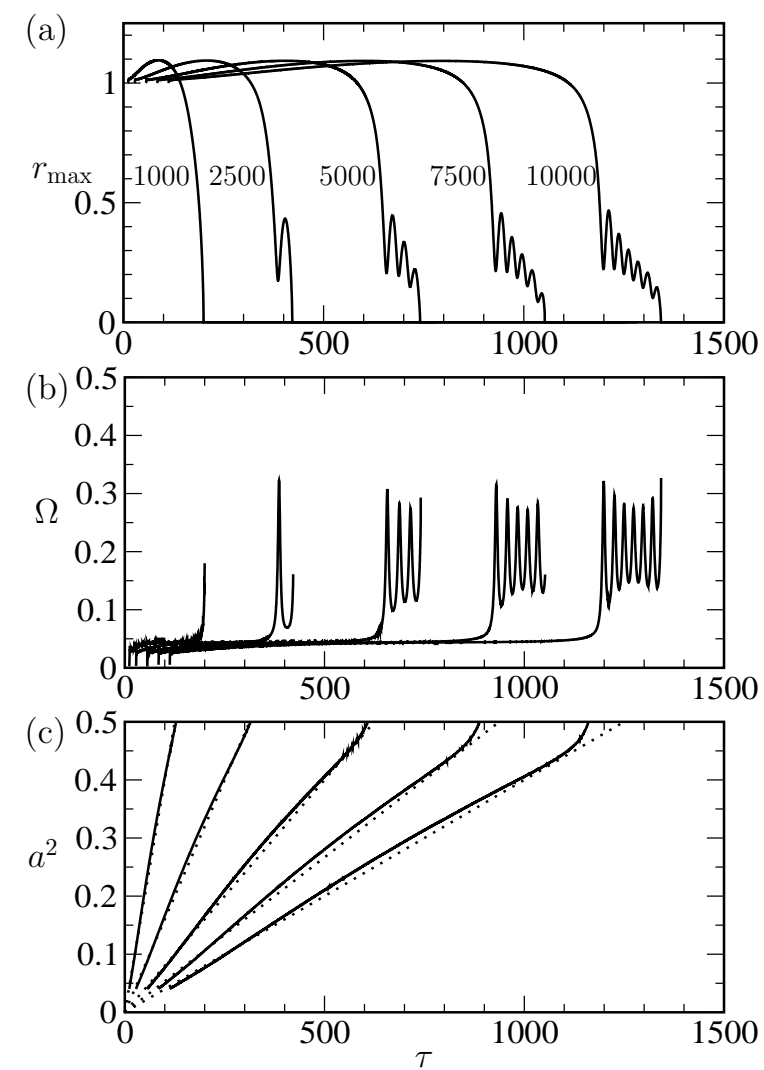

Figure 5: Large pitch $L=2$. Influence of the Reynolds number (Re values are indicated on the figures) on (a) the radial position $r_{\text {max }}$ of vorticity maximum versus time $\tau$; (b) the angular velocity $\Omega(\tau)$ of the vorticity maximum; (c) the square of the core size $a^{2}(\tau)$ (the dotted line corresponds to the 2D diffusion law $a^{2}(\tau)=4 \tau / R e$ ).

(figure 7b). This reduced rotating speed for the vortex pair originates from the increasing role of the selfinduced motion of each vortex. For a straight vortex $(L=\infty)$, the self-induced velocity vanishes; in presence of curvature (finite $L$ ), the self-induced velocity tends to make each vortex rotate clockwise [10]. This effect thus counterbalances the mutual interaction i.e. the one induced by the companion vortex, which is always present. The frequency $\omega_{3}$ of oscillations during phase 3 tends to zero when $L$ is decreased. It can be fitted as a function of $1 / L$ (figure $8 \mathrm{k}$ ): $\omega_{3}^{2} \sim 0.93\left(L_{\mathrm{c}}^{-1}-L^{-1}\right)$ with $L_{\mathrm{c}}=1.82$. The pitch $L_{c}$ provides a well-defined threshold value which separates the merging dynamics at large pitches from a different one at intermediate pitches. The durations of phase 1,2 and 3 all scale as powers of $\left(L_{\mathrm{c}}^{-1}-L^{-1}\right)$ (see figure $8 \mathrm{~b}$ ). By contrast, the core size is relatively unchanged by $L$ (figure 7 f). 

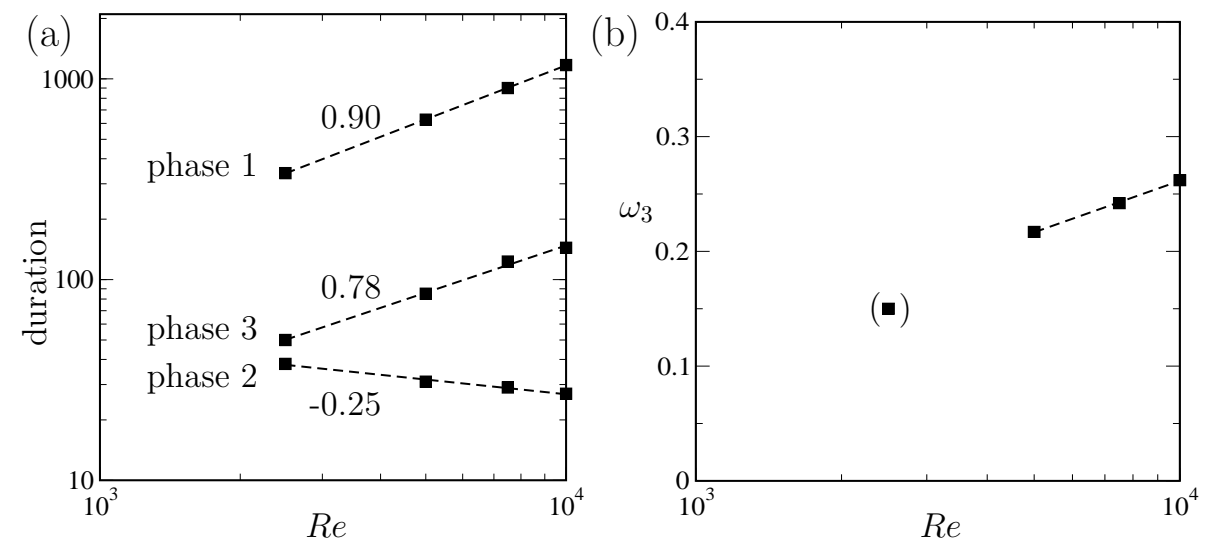

Figure 6: Large pitch $L=2$. (a) Influence of the Reynolds number $R e$ on the duration of phases 1,2 and 3. A power law is obtained for each phase with an exponent written on the graph. (b) Oscillation frequency $\omega_{3}$ during phase 3 as a function of $R e$. The bracketted value is based on only one oscillation. The dashed line corresponds to the scaling law $\omega_{3} \sim C+\log R e^{0.064}$.

\subsection{Quasi-steady state before merging and merging onset}

During phase 1, there exists a univoque dependency (see figure 9 between $\alpha \omega_{B}$ or $u_{H}$ and $\psi_{R}$, the streamfunction defined in the frame of reference rotating with the vortices i.e.

$$
\psi_{R}=\psi+\frac{r^{2}}{2} \Omega
$$

This feature is related to the presence of a quasi-equilibrium. For an inviscid dynamics, an equilibrium solution rotating with a constant angular velocity $\Omega$ exists. It has been shown [5] that, for this solution, velocity $u_{H}(r, \varphi-\Omega t)$ is a univoque function of $\psi_{R}$ :

$$
u_{H}=F\left(\psi_{R}\right) \text {. }
$$

Most theoretical studies are based on this inviscid approximation with $u_{H}=0$. In that even more specific case, $\alpha \omega_{B}$ is also a univoque function of $\psi_{R}$ :

$$
\alpha \omega_{B}=G\left(\psi_{R}\right) .
$$

Both conditions are satisfied here in a viscous context: during phase 1, the flow thus follows an inviscid quasi-equilibrium (see figure 9 ). However, the vortex core size, angular velocity and distribution evolve 

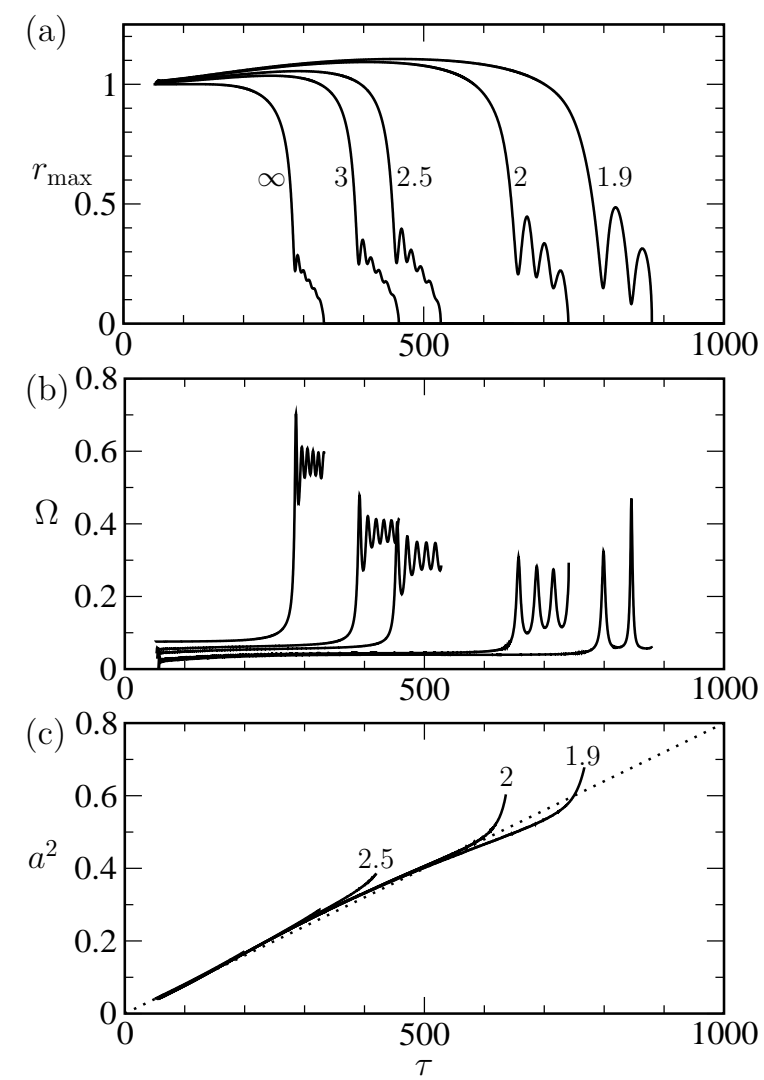

Figure 7: Large pitch. Influence of $L(L=\infty, 3,2.5,2,1.9)$ for $R e=5000$ on (a) the radial position $r_{\text {max }}$ of the vorticity maximum versus time $\tau$; (b) the angular velocity $\Omega(\tau)$ of the vorticity maximum; (c) the square of the core size $a^{2}(\tau)$ (the dotted line corresponds to the $2 \mathrm{D}$ diffusion law).

through viscous diffusion. It can be demonstrated 1 in a viscous context, that, if $u_{H}(\tau=0)=0$, then $u_{H}$ evolves in time according to:

$$
u_{H}=-\frac{2 \tau}{L R e} \alpha \omega_{B}
$$

This relation is also verified in the viscous simulation (see figure $9 \mathrm{~b}$ ). Note that, since $t=0$ corresponds to $\tau=-t_{\star}$, we use the following initial $u_{H}$ distribution for the simulations:

$$
u_{H}(r, \varphi, t=0)=\frac{2 t_{\star}}{L R e} \alpha \omega_{B}
$$

\footnotetext{
${ }^{1}$ A paper presenting the proof of relation 11 is about to be submitted.
} 

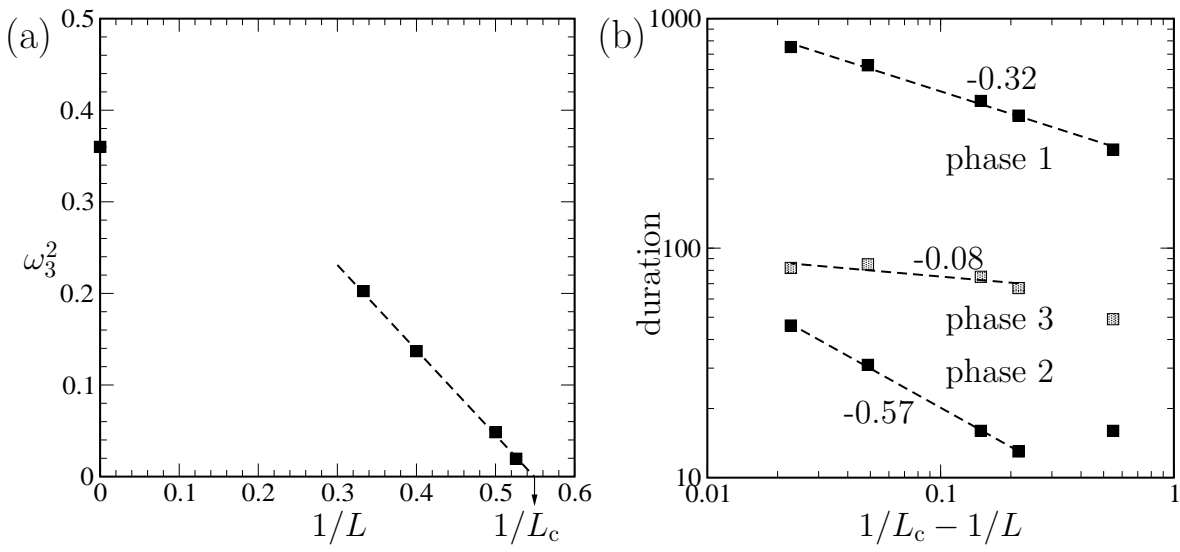

Figure 8: Large pitch. (a) Square of the oscillation frequency $\omega_{3}$ during phase 3 as a function of $1 / L$. (b) Duration of phases 1,2 and 3 as a function of $L_{\mathrm{c}}^{-1}-L^{-1}$. A power law is obtained with the exponent written in the graph.
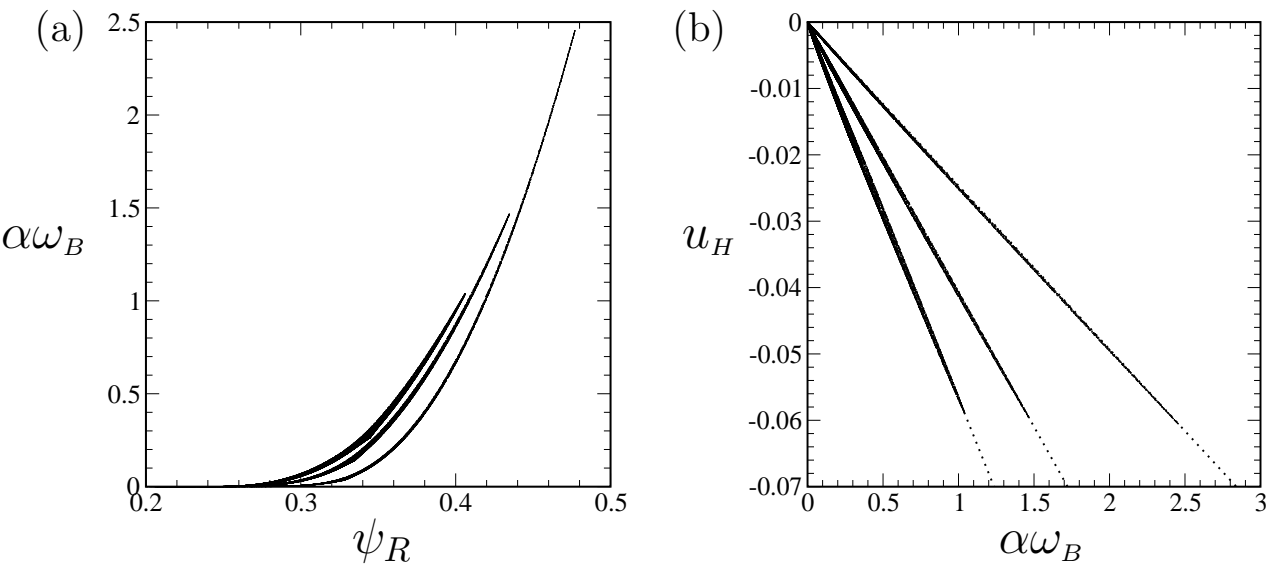

Figure 9: Simulation for $L=2.5$ and $\operatorname{Re}=5000$ for $\tau=154,254,354$ (the curves move from right to left with increasing time $\tau$ ). (a) Scatter plots for $\alpha \omega_{B}$ vs $\psi_{R}$; (b) Scatter plots $u_{H}$ vs $\alpha \omega_{B}$ (the dotted line corresponds to the analytical formula (11).

The maximum absolute value of $u_{H}$ is thus initially small and remains so as time evolves: this weak jet component emerging through viscous effects is not thought to play any active dynamical role in the present context. Higher initial values of $u_{H}$ corresponding to helical vortices with swirl are left for future studies.

In the two-dimensional case, phase 1 - and thus the merging onset - is associated to the existence of the above quasi-equilibrium state and/or its stability. Quantitatively, phase 1 persists until $a(\tau) / r_{\max }(\tau)$ exceeds a threshold value [11]. For the helical case, the merging is delayed as $1 / L$ is increased. Paradoxically, for a given time $\tau$ in phase $1, r_{\max }(\tau)$ slightly increases with increasing $1 / L$ (figure $7 \mathrm{~h}$ ) and the vortex core 
(a)

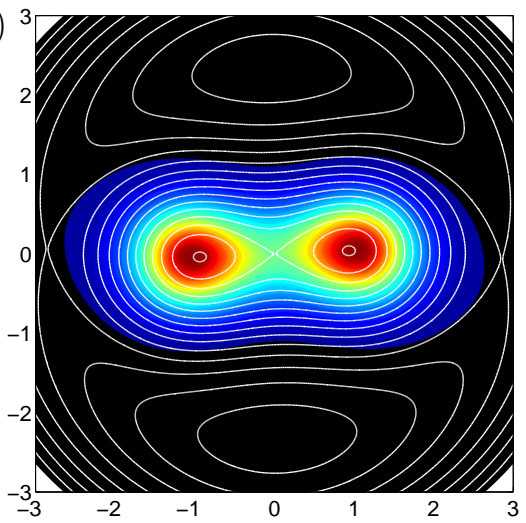

(b)

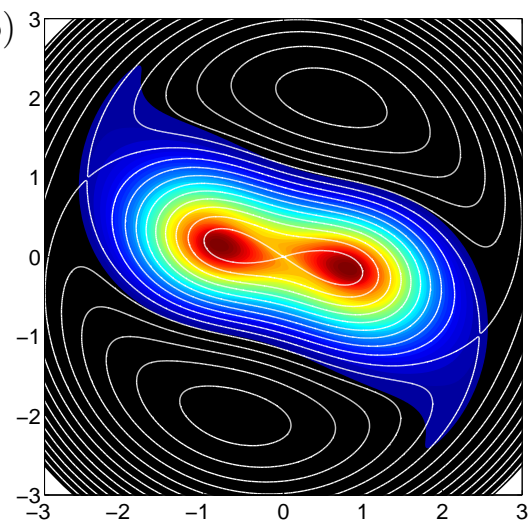

Figure 10: Isocontours of vorticity component $\omega_{B}$ (colored and filled) and streamlines of $\psi_{R}$ (white lines) for $L=2$ at $R e=5000$ (a) before critical time $\tau_{1}(\mathrm{~b})$ just after critical time $\tau_{1}$.

(a)

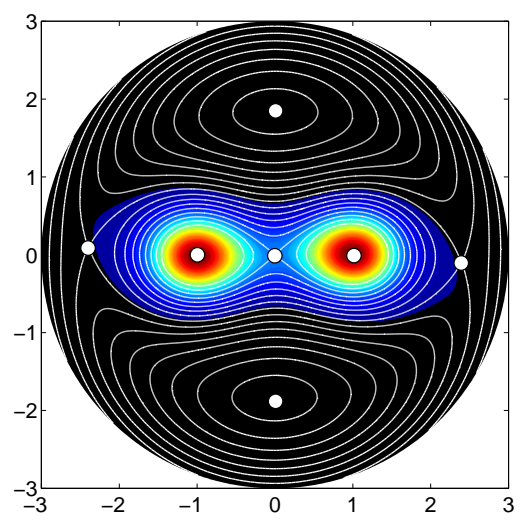

(b)

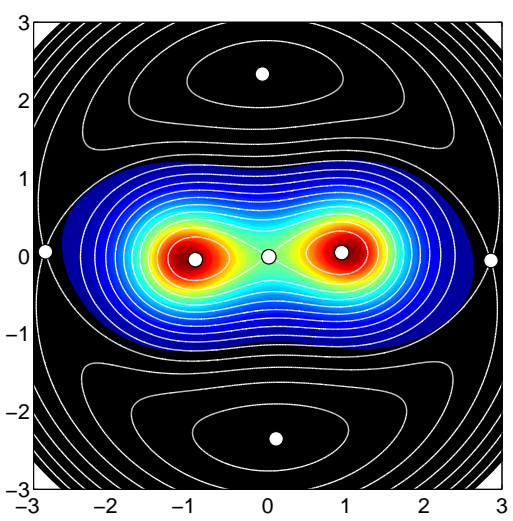

Figure 11: Isocontours of vorticity component $\omega_{B}$ (colored and filled) and streamlines of $\psi_{R}$ (white lines) for (a) $L=3$ and (b) $L=2$ at $R e=5000$ near critical time $\tau_{1}$ before the convective phase 2. The streamline pattern displays two types of hyperbolic points and two types of elliptic points. Note that the same scale is used for both graphes, although the sizes of the computational domain are (a) $R_{\mathrm{ext}}=3$ and (b) $R_{\mathrm{ext}}=4$.

size does not change with $1 / L$ (see figure 7 r) remaining close to the two-dimensional law $a^{2}(\tau)=4 \tau / R e$. Yet, the critical core size $a\left(\tau_{1}\right)$ at which phase 2 begins, increases as $1 / L$ is increased. An explanation can be found by recalling the way the two-dimensional merging occurs for identical vortices: the convective merging phase 2 begins when a significant amount of vorticity has escaped the closed co-rotating streamlines of the two vortices, and begins to form filaments in the surrounding fluid [12, 13, 14]. A similar scenario takes place here, as shown on figure 10, at critical time $\tau_{1}$, vorticity has filled the atmosphere of the two vortices, and begins to escape into the peripheral rotating fluid through the external hyperbolic points of the 
co-rotating streamfunction, because of viscous diffusion. The subsequent formation of filaments is believed to be associated to the convective phase 2 whereby vortices are radially pushed towards the axis. In figure 11 , the hyperbolic points move towards larger $r$ as soon as $L$ is decreased. The critical core size thus increases together with $d$ as $L$ is decreased, which makes phase 1 last longer.
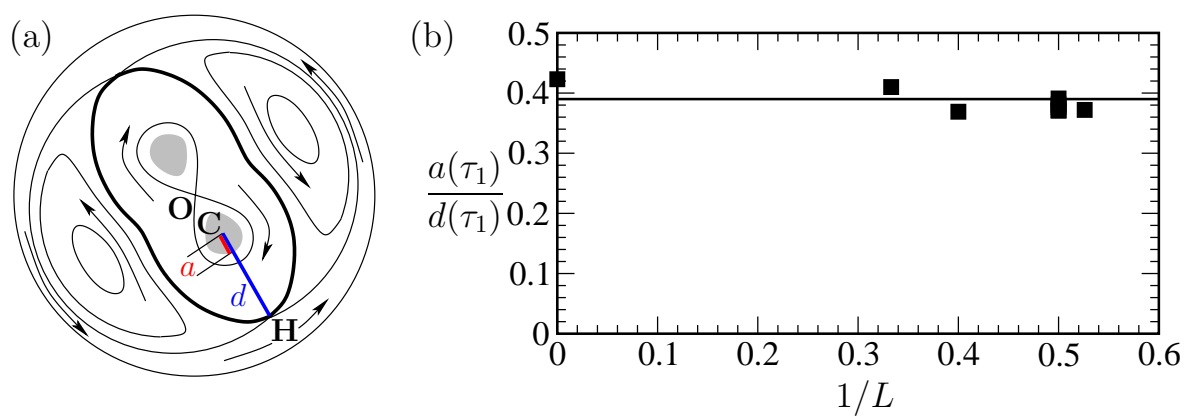

Figure 12: (a) Definition of the distance $d$ between vorticity maximum and the outer hyperbolic point of the corotating streamfunction $\psi_{R}$. (b) Critical value $a / d\left(\tau_{1}\right)$ as a function of $1 / L$.

For different values of $L$ and $R e$, the pertinent parameter for convective merging is not the ratio $a / r_{\max }$, but rather the ratio between the core size $a$ and the distance $d$ between the vortex center and the outer hyperbolic point (see figure $12 \mathrm{k}$ ). For the two-dimensional case, $r_{\max }$ and $d$ are clearly related. As $1 / L$ is increased, the rotation speed decreases thus causing the hyperbolic points of the co-rotating streamfunction to move away from the axis, i.e. length $d$ increases. It is observed from the simulations that, at critical time, the ratio $a / d\left(\tau_{1}\right)$ is almost constant and equals 0.39 (see figure 12b)). This value is weakly dependent on the Reynolds number: for instance, at $1 / L=0.5$ on figure $12 \mathrm{~b}$, the values $a / d\left(\tau_{1}\right)$ are plotted for several Reynolds numbers.

\section{Diffusive merging at intermediate pitch $1.1 \leq L \leq 1.8$}

As the helical pitch is decreased, the angular velocity computed in the DNS decreases and eventually becomes negative. The zone of intermediate pitch $1.1 \leq L \leq 1.8$ is thus characterised by weak vortex rotation, and the outer hyperbolic point is pushed away far from the axis, or does not exist at all. In that instance, neither filamentation nor convective merging can occur, and the behaviour of the system is dominated by viscous diffusion which yields a slow, smooth merging process (figure 13). Actually, there exist two types of diffusive merging depending whether $L$ is larger or smaller than $L_{d} \approx 1.25$, corresponding to two different 
(a)

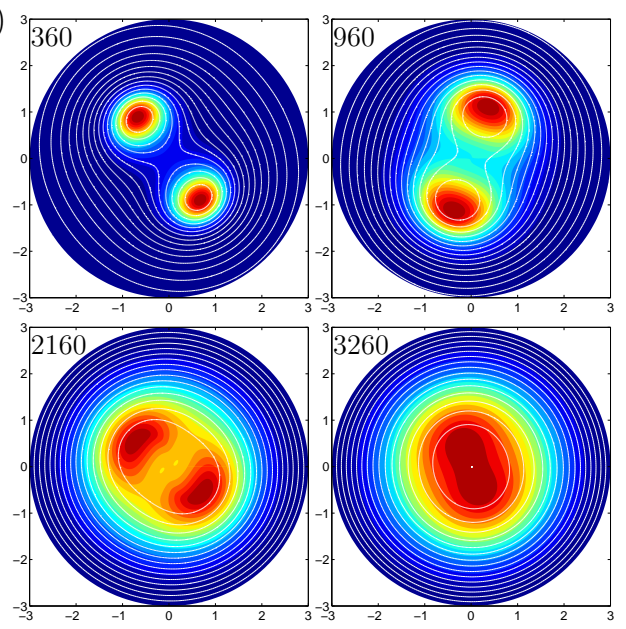

(b)

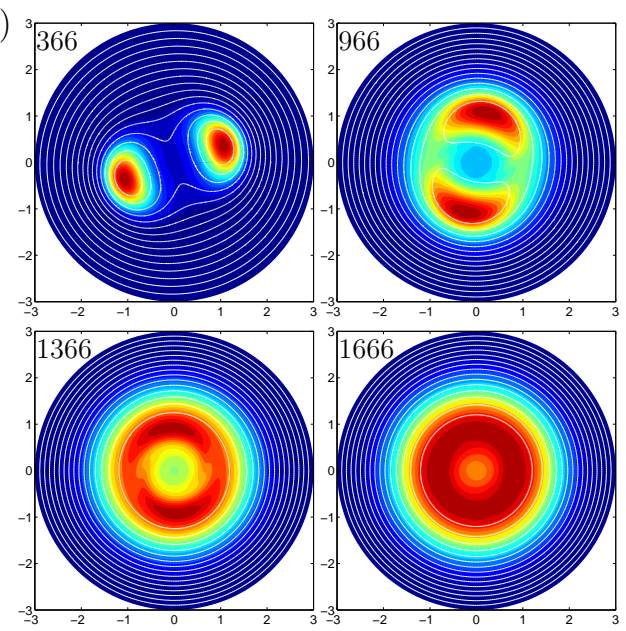

Figure 13: Intermediate pitch and $R e=5000$. Isocontours of vorticity component $\omega_{B}$ (colored and filled) and $\psi_{R}$ (white lines). (a) $L=1.5$ at times $\tau=360,960,2160,3260$. (b) $L=1.2$ at time $\tau=366,966,1366,1666$.

(a)

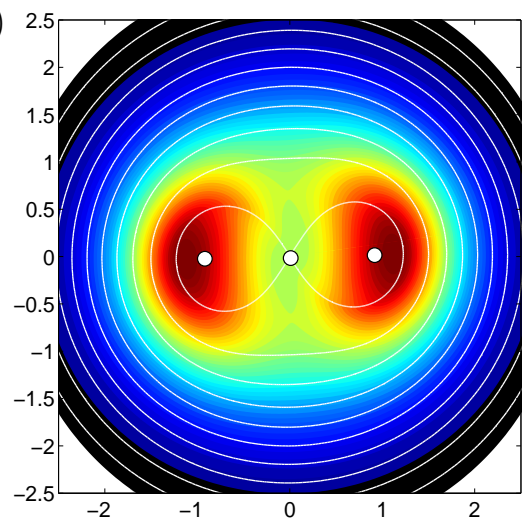

(b)

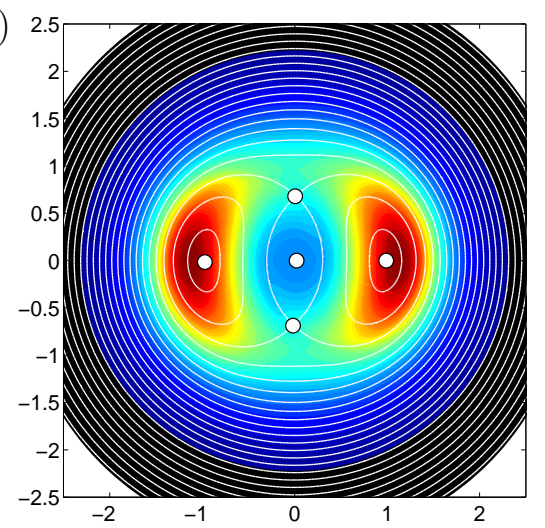

Figure 14: Intermediate pitch and $R e=5000$. Isocontours of $\omega_{B}$ (colored and filled) and $\psi_{R}$ (white lines) and position of elliptic and hyperbolic points. (a) $L=1.5$ at $\tau=1561$. (b) $L=1.2$ at $\tau=866$.

topologies of the streamlines in the co-rotating frame. For a first one $L \geq L_{d}$ (see $L=1.5$ case in figure 14a), the merging takes place at the axis, whereas for a second one $L \leq L_{d}$ (see $L=1.2$ case in figure 14b), the merging takes place at a radial distance of order unity and forms an axisymmetric corona of vorticity. In both cases, the flow keeps the initial central symmetry.

For case $L \geq L_{d}$, an hyperbolic point for the streamlines is present at the center. On the contrary, for $L \leq L_{d}$, the center becomes elliptic and two hyperbolic points emerge away from the center, thus separating 
the central region and explaining why the vorticity concentrates along a corona. As a matter of fact, this latter merging occurs between coils rather than at the axis. For both cases, the flow is dominated by the diffusion effects, as shown by plotting the radial position $r_{\max }$, angular velocity $\Omega$ and core size $a^{2}$ as functions of a rescaled time $\tau / \operatorname{Re}$ (figure 15). Note however that the vortex core size now significantly departs from the two-dimensional viscous diffusion law.
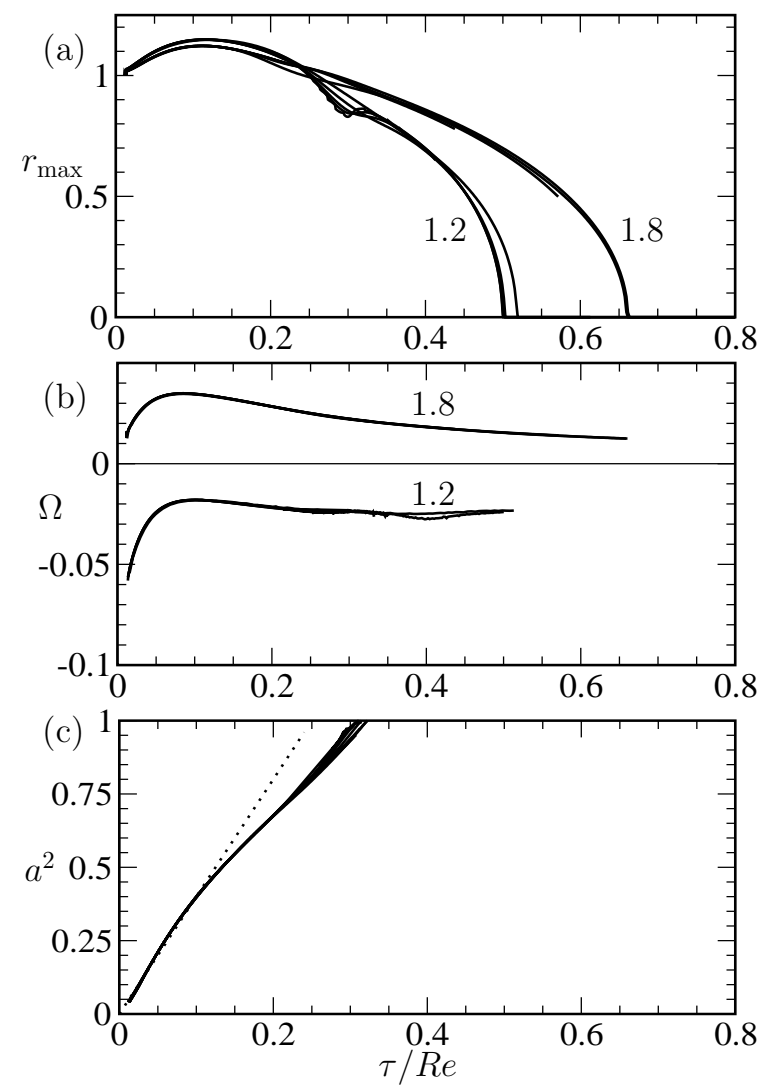

Figure 15: Intermediate pitch $L=1.8$ and $L=1.2$. For a given $L$, the curves at various Reynolds numbers $(R e=$ $1000,2500,5000,7500,10000)$ collapse when displayed in terms of $\tau / R e$. (a) Radial position $r_{\max }(\tau / R e)$ of the vorticity maximum; (b) angular velocity $\Omega(\tau / R e)$ of the vorticity maximum; (c) square of the core size $a^{2}(\tau / R e)$ (the dotted line represents the 2D diffusion law).

\section{Small pitch vortices $L \leq 1.1$ : Okulov's instability}

In the simulation at $L=1$ displayed in figure 16, the system evolves similarly to the case of diffusive merging. However if this simulation is started with an initial perturbation (one vortex radially pushed by 
(a)

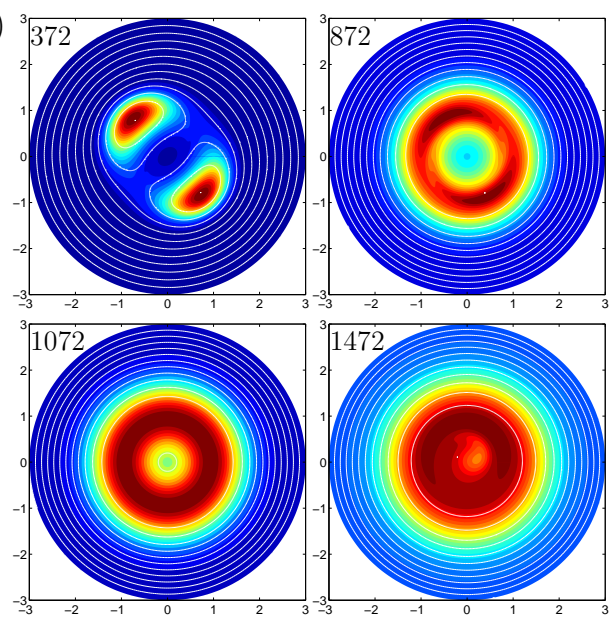

(b)

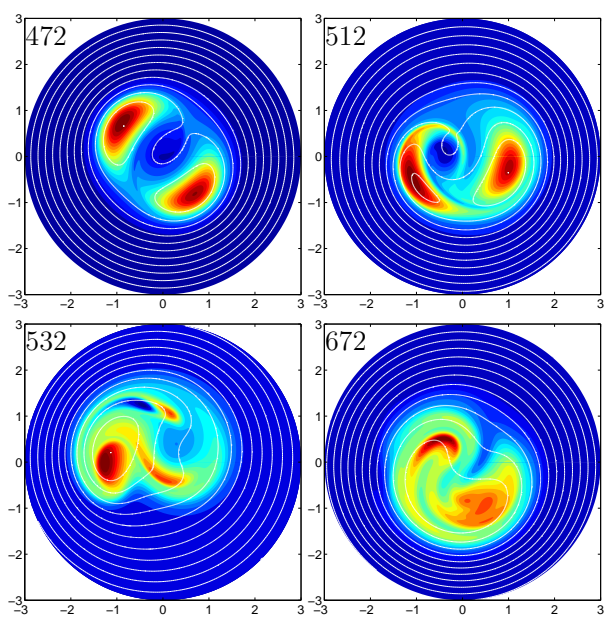

Figure 16: Small pitch $L=1$ and $R e=5000$. (a) Isocontours of vorticity component $\omega_{B}$ (colored and filled) and $\psi_{R}$ (white lines) at $\tau=372,872,1072,1472$. Here simulations are initiated without perturbation. (b) Isocontours of $\omega_{B}$ at $\tau=472,512,532,672$. Here, simulation starts from a state in which vortex positions have been moved by an amount 0.001 .

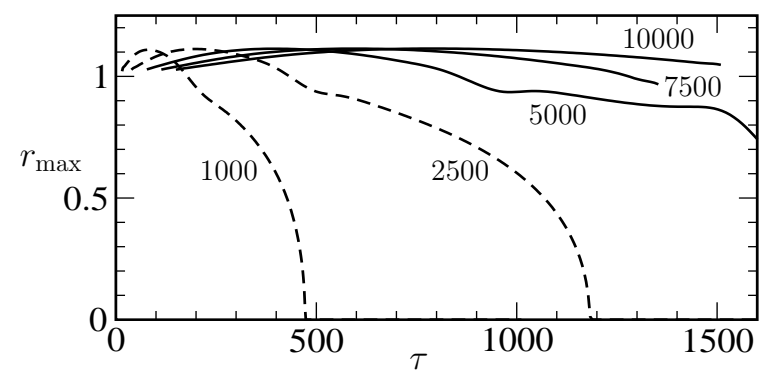

Figure 17: Small pitch $L=1$. Influence of the Reynolds number on the radial position $r_{\max }$ of the vorticity maximum versus time $\tau$. Here simulations are initiated without perturbation. Dashed lines correspond to an evolution into an axisymmetric state and continuous lines to an evolution dominated by symmetry-breaking instability. The case $R e=5000$ is particular: it reaches an axisymmetric state but subsequent symmetry breaking instability.

0.001), the resulting evolution (see figure $16 \mathrm{~b}$ ) is quite different: an instability mechanism breaking the central symmetry between vortices is active, and its effects are clearly felt at $\tau=472$. Indeed, at small pitches, a helical vortex pair is known to be unstable [15] for small core size filaments with respect to displacement modes, a phenomenon responsible for the destabilisation of propeller and wind turbine wakes. The critical pitch under which such instability occurs for a vortex pair in the limit of small core sizes is $L_{i}=1.106$. The present simulation indicates that this instability is not altered by finite core size.

Predicting precise frontiers between the diffusive and instability regimes is not an easy task since the 
instability growth rate depend on the vortex core size, which is a time-dependent quantity in viscous simulations. A closer inspection to the simulation started without initial perturbation (actually with a perturbation comparable to numerical precision) shows that the system forms a corona of vorticity through viscous diffusion before the instability becomes observable (see $\tau=1072$ in figure 16a). Hence for the lowest Reynolds numbers, the dynamics is essentially a diffusive merging process. Eventually however, it may happen that the instability becomes visible and slightly breaks the axisymmetry of the corona (snapshot at $\tau=1472$ ). The influence of the Reynolds number is shown in figure 17

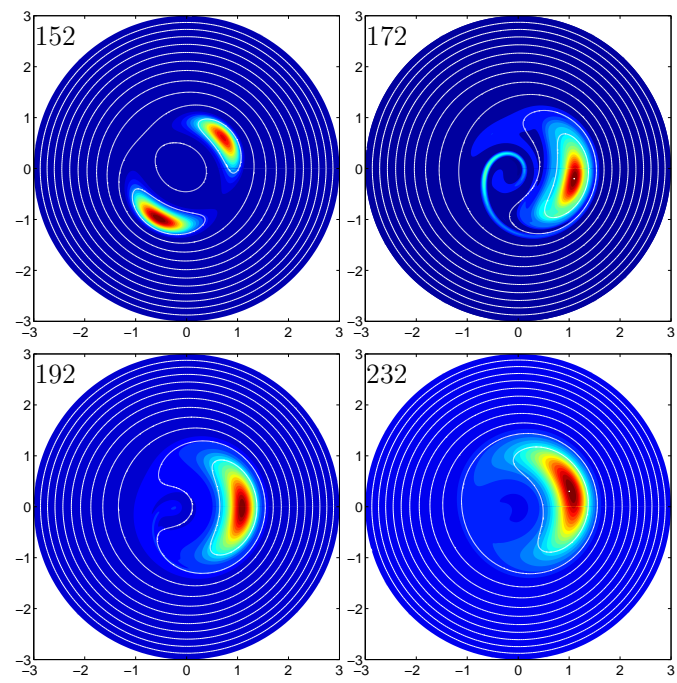

Figure 18: Simulation for $L=0.5$ and $R e=5000$, starting from a state in which the vortex positions have been perturbed by an amount 0.001 . Isocontours of vorticity component $\omega_{B}$ (colored and filled) and $\psi_{R}$ (white lines) at $\tau=152,172,192,232$.

In the simulations at lower pitches such as $L=0.5$ (see figure 18), the growth rate of Okulov's instability is more important and the system gets destabilised more rapidly: one helical vortex is strongly stretched (see at $\tau=172)$ and merges with the other $(\tau=192)$. It is remarkable that this behaviour reminiscent of the vortex grouping observed in some experiments $[1,16]$ can be described in the present helically symmetric framework. The present study essentially dealt with helical vortices initially located at $\theta=\theta_{0}$ and $\theta_{0}+\phi$ with $\phi \approx \pi$. However, the numerical code is also able to tackle cases with $\phi$ quite different from $\pi$. Some runs were performed for such cases and led to merging dynamics similar to those presented in figure 18 


\section{Conclusion}

We numerically investigated the dynamics of a pair of identical helical viscous vortices with respect to their helical pitch and Reynolds number. At large pitch, vortex merging occurs that is analogous with the two-dimensional vortex merging: its underlying mechanism is due to the emission of vorticity filaments. In addition, a threshold for merging can be defined based on the external hyperbolic point position. At intermediate pitch 1.1. $\leq L \leq 1.8$, the system rotates slowly around the axis, and diffusive effects dominate the dynamics. At smaller pitch $L \leq 1.1$, a slight shift of the initial vortex positions is able to rapidly destabilize the system leading to vortex grouping. The different types of merging in the $(R e, L)$ plane are displayed on figure 19 Similar trends have been found for the three-helical vortex system [17]. In a future work, the influence of a hub vortex or of initial velocity $u_{H}$ both present in practical simulations and experiments will be investigated and their role in the helical vortex dynamics will be determined. Furthermore, quasi-equilibrium rotating states obtained and described herein will define precise basic states to be used in a general linear stability analysis. In that framework, initial three-dimensional perturbations breaking the helical symmetry will be considered: this work will be hence performed in the spirit of Refs. [18, 19], but in a viscous linear or nonlinear context.

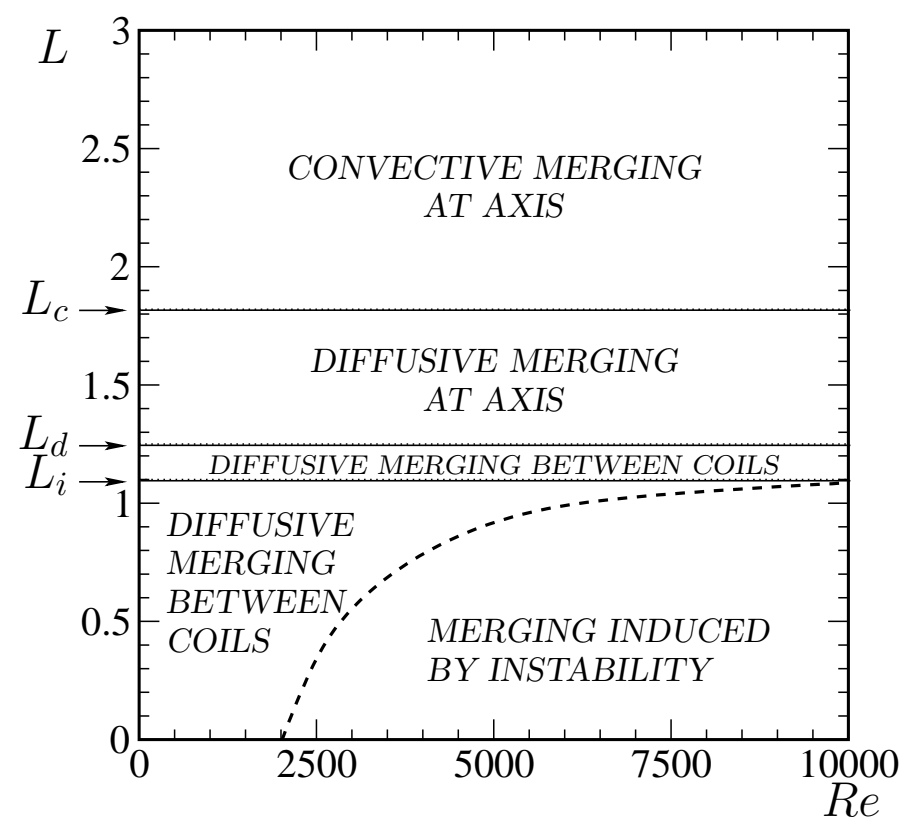

Figure 19: Types of merging for two helical vortices, in the $(R e, L)$ plane. 


\section{Acknowledgments}

This article is dedicated to our friend and collegue Patrick Huerre with whom two of us have been collaborating for quite a while. He was our cicerone along steep pathes in the green valley of open flow

[6] W.J. Devenport, C.M. Vogel, J.S. Zoldos, Flow structure produced by the interaction and merger of a pair of co-rotating wing-tip vortices, J. Fluid Mech. 394 (1999) 357-377.

[7] I. Delbende, M. Rossi, O. Daube, DNS of flows with helical symmetry, Theor. Comput. Fluid Dynam. 26(1) (2012) 141-160.

255

[8] B.Piton, Simulations de tourbillons à symétrie hélicoïdale, Ph.D thesis, Sorbonne Universités, UPMC Univ Paris 06, 2011.

[9] P.G. Saffman, Vortex Dynamics, Cambridge University Press, Cambridge, 1992. 
[10] S.V. Alekseenko, P.A. Kuibin, V.L. Okulov, Theory of Concentrated Vortices: An Introduction, Springer-Verlag, Berlin, 2007.

260

[19] B.P. Gupta, R.G. Loewy, Theoretical analysis of the aerodynamic stability of multiple, interdigitated helical vortices, AIAA J. 12(10) (1974) 1381-1387. 